\title{
Rodent Progressive Cardiomyopathy
}

National Cancer Institute

\section{Source}

National Cancer Institute. Rodent Progressive Cardiomyopathy. NCI Thesaurus. Code C161561.

A spontaneous, age-related cardiac disease of rats and mice, characterized by myocardial changes presenting a continuum that begins as focal to multifocal individual cardiomyocyte necrosis attended by a few inflammatory cells prog ressing at different rates in different animals to include multifocal mononuclear cell inflammation and even fibrosis for larger lesions. (INHAND) 